\title{
Novel Photocatalytic Function of Porphyrin-Modified Gold Nanoclusters in Comparison with the Reference Porphyrin Compound
}

\section{Derivation of eq 3.}

The rates of formation of $\mathrm{HV}^{\bullet+},{ }^{1} \mathrm{H}_{2} \mathrm{P}^{*}$, and ${ }^{3} \mathrm{H}_{2} \mathrm{P}^{*}$ are given by eqs $\mathrm{S} 1-\mathrm{S} 3$, respectively, where In is the light intensity absorbed by $\mathrm{H}_{2} \mathrm{P}$.

$$
\begin{aligned}
& \mathrm{d}\left[\mathrm{HV}^{\bullet+}\right] / \mathrm{dt}=2 k_{\text {et }}^{\mathrm{S}}\left[{ }^{1} \mathrm{H}_{2} \mathrm{P}^{*}\right]\left[\mathrm{HV}^{2+}\right]+2 k^{\mathrm{T}}{ }_{\text {et }}\left[{ }^{3} \mathrm{H}_{2} \mathrm{P}^{*}\right]\left[\mathrm{HV}^{2+}\right] \\
& \mathrm{d}\left[{ }^{1} \mathrm{H}_{2} \mathrm{P}^{*}\right] / \mathrm{dt}=I n-t_{\mathrm{f}}^{-1}\left[{ }^{1} \mathrm{H}_{2} \mathrm{P}^{*}\right]-k_{\mathrm{ISC}}\left[{ }^{1} \mathrm{H}_{2} \mathrm{P}^{*}\right]-k^{\mathrm{S}}{ }_{\mathrm{et}}\left[{ }^{1} \mathrm{H}_{2} \mathrm{P}^{*}\right]\left[\mathrm{HV}^{2+}\right] \\
& \mathrm{d}\left[{ }^{3} \mathrm{H}_{2} \mathrm{P}^{*}\right] / \mathrm{dt}=k_{\mathrm{ISC}}\left[{ }^{1} \mathrm{H}_{2} \mathrm{P}^{*}\right]-t_{\mathrm{T}}^{-1}\left[{ }^{3} \mathrm{H}_{2} \mathrm{P}^{*}\right]-k^{\mathrm{T}}{ }_{\mathrm{et}}\left[{ }^{3} \mathrm{H}_{2} \mathrm{P}^{*}\right]\left[\mathrm{HV}^{2+}\right]
\end{aligned}
$$

Under the steady state conditions, $\left[{ }^{1} \mathrm{H}_{2} \mathrm{P}^{*}\right]$ and $\left[{ }^{3} \mathrm{H}_{2} \mathrm{P}^{*}\right]$ are given by eqs $\mathrm{S} 4$ and $\mathrm{S} 5$, respectively.

$$
\begin{aligned}
& {\left[{ }^{1} \mathrm{H}_{2} \mathrm{P}^{*}\right]=\operatorname{In} /\left(t_{\mathrm{f}}^{-1}+k_{\mathrm{ISC}}+k^{\mathrm{S}}{ }_{\mathrm{et}}\left[\mathrm{HV}^{2+}\right]\right)} \\
& {\left[{ }^{3} \mathrm{H}_{2} \mathrm{P}^{*}\right]=k_{\mathrm{ISC}} \operatorname{In} /\left(t_{\mathrm{T}}^{-1}+k^{\mathrm{T}}{ }_{\mathrm{et}}\left[\mathrm{HV}^{2+}\right]\right)\left(t_{\mathrm{f}}^{-1}+k_{\mathrm{ISC}}+k^{\mathrm{S}}{ }_{\mathrm{et}}\left[\mathrm{HV}^{2+}\right]\right)}
\end{aligned}
$$

By substituting eqs $\mathrm{S} 4$ and $\mathrm{S} 5$ into eq $\mathrm{S} 1, \Phi_{\mathrm{obs}}\left[=\left(\mathrm{d}\left[\mathrm{HV}^{\bullet+}\right] / \mathrm{dt}\right) / \mathrm{In}\right]$ is given by eq 3 . 